\title{
Los payasos como herramienta socioeducativa para la intervención comunitaria ${ }^{1}$ \\ Clowns as an social and educative tool for community Intervention
}

\author{
Ainhoa Gana-Dañobeitia* \\ Asier Huegun-Burgos** \\ Itziar Rekalde-Rodríguez ${ }^{* * *}$
}

\section{Resumen}

El valor de la disciplina artística del payaso social desde su función política, antropológica y social, ha sido estudiada estos últimos años (Domínguez, 2015; Fo, 2008; Rico-Medina, 2015), pero no así la formación y mediación en el impacto del payaso socioeducativo (Ros-Clemente, 2015b). De ahí que este trabajo tenga por finalidad el profundizar en esta modalidad de payaso y desvelar las capacidades y posibilidades que esta disciplina posee como herramienta educativa. La investigación se centra en

${ }^{1}$ Este estudio es fruto del trabajo de investigación original titulado Pailazogintza soziohezitzailea: praktika pedagogiko komunitarioa, llevado a cabo en el curso 2017/18 en el marco del Máster universitario en Investigación en Ambitos Socioeducativos de la Escuela de Máster y Doctorado de la Universidad del País Vasco/Euskal Herriko Unibertsitatea, y avalado por el Departamento de Didáctica y Organización escolar de la Facultad de Educación, Filosofía y Antropología de la UPV/EHU.

* Máster Universitario en Investigación en Ambitos Socioeducativos e Investigadora predoctoral de la Universidad del País Vasco/Euskal Herriko Unibertsitatea. Lejona, España. Correo electrónico: ainhoagana@gmail.com.

** Licenciado en pedagogía, Doctor en ciencias de la educación y Profesor del Departamento de Didáctica y Organización escolar de la Facultad de Educación, Filosofía y Antropología de la Universidad del País Vasco/Euskal Herriko Unibertsitatea. Lejona, España. Correo electrónico: asier.huegun@ehu.eus.

*** Licenciada en Pedagogía de la Universidad de Salamanca, Máster en la Unión Europea y Derechos Humanos de la Universidad Pontificia de Salamanca y Programa de Doctorado en Historia de la Educación de la Universidad de Salamanca. Profesora del Departamento de Didáctica y Organización escolar de la Facultad de Educación, Filosofía y Antropología de la Universidad del País Vasco/Euskal Herriko Unibertsitatea. Lejona, España. Correo electrónico: asier.huegun@ehu.eus. Correo electrónico: itziar.rekalde@ehu.eus.

Universidad del Valle. Cali, Colombia. Recibido: 25/07/2018 Aprobado: 13/11/2018 ISSN: 0122-1213 ISSN-e: 2389-993X Doi: 10.25100/prts.v0i27.6733] 
un estudio de caso, donde el caso lo constituye el grupo de payasos del País Vasco Takolo, Pirritx, Porrotx y Marimotots. Se han utilizado entrevistas individuales en profundidad con cada uno de los participantes, un grupo de discusión con todos ellos y análisis documental de sus materiales y producciones artísticas. El sistema categorial resultante del procedimiento analítico ha ayudado a revelar que son los valores universales de respeto, igualdad y solidaridad, entre otros, los que intentan transmitir vehiculizándolos a través de una lengua minoritaria cuyo objetivo es también impulsarla. Se concluye con las características del modelo de intervención asentado en los principios de la educación inclusiva que han desarrollado el referido grupo de payasos.

Palabras clave: Payaso social; Desarrollo comunitario; Lenguas minoritarias; Inclusión; Intervención socioeducativa.

\section{Abstract}

The value of the artistic discipline of the social clown from its political, anthropological and social function, has been studied in recent years (Domínguez, 2015; Fo, 2008; Rico-Medina, 2015), but the same is not true for the training of the socio-educational clown and their impact on society (Ros-Clemente, 2015b). As a result, this study aims to delve into this mode of clowning and uncover the capabilities and possibilities that this discipline has as an educational tool. The research focuses on a case study, where the case is a group of clowns from the Basque Country named Takolo, Pirritx, Porrotx and Marimotots. The instruments used consist of individual interviews with each of the participants, a focus group with all of them and finally, documentary analysis of their materials and artistic productions. The categorical system resulting from the analytical procedure has helped to reveal that the clowns transmit universal values such as respect, equality and solidarity, among others, and that they do so through a minority language, thereby promoting its use. The paper concludes by stating the characteristics of the model of intervention, which is based on the principles of inclusive education developed by the group of clowns.

Keywords: Social clown; Community development; Minority languages; Inclusion, socio-educational intervention. 
Sumario: 1. Introducción; 2. La trayectoria de los payasos vascos en las últimas décadas; 3. Objetivos de investigación; 4. Metodología; 5. Resultados e interpretación; 6. Conclusiones; 7. Referentes.

\section{Introducción}

Detallar la perspectiva histórica sobre el payaso y su evolución nos ayudará a situar las fuentes teóricas del trabajo. Según diferentes publicaciones, el término clown se utiliza como término universal para definir esta disciplina artística (Nicoll, 1977; Crowther, 1979; SáinzMoreno, 1990). Sin embargo, no hay un acuerdo sobre la diferencia entre los términos payaso y clown que participan de este oficio (Ros-Clemente, 2015a). Por lo tanto, como son muchas las clases de payasos y las habilidades que realizan ¿de qué estamos hablando cuando hacemos uso del término payaso?

Históricamente, el payaso ha tenido un objetivo simple y claro: divertir, entretener y hacer reir (Jara, 2011). No obstante, no hay ningún texto que explique la completa definión del payaso, ya que en la historia ha habido payasos de todo tipo en el mundo: arlequín, saltimbanqui, bufón, oliver, augusto, contraugusto, cara-blanca, pierrot, polichinela, tony, cómico, trovador, tramp, titiritero, volatinero, mimo, mesie loyal, clown o juglar ..., en cambio el comportamiento de ellos en sus espectáculos ha sido diferente. Por lo tanto, cuando hablamos de todos estos términos, nos estamos refieriendo a los payasos. Esto quiere decir que hablamos del payaso (tomando en consideración todas sus variedades) cuando hablamos de la personas que comparten su forma de ver el mundo en clave de humor, con el objetivo de remover emociones.

En cualquier caso, el payaso oficialmente no ha sido visto con buenos ojos: al igual que se ha visto como ridículo y tramposo, ha sido menospreciado, censurado y marginado en muchas épocas. De todos modos, como recalca Fo (2008), el trabajo de los payasos no es baladí, ya que se considera como un quehacer constante, disciplinado y que requiere de mucho esfuerzo; además, Rico-Medina (2015) valora la función social, 
política y antropológica del mismo. No obstante, Fo (2008) cree que el payaso ha perdido su compromiso y su influencia política.

Ceballos (1999) se toma muy en serio el potencial cómico del payaso, ya que concluye que el payaso tiene la capacidad de influir en las emociones de las personas valiéndose de todo su cuerpo. Además, como menciona Domínguez (2015), el payaso ha subido muchos escalones en todo el mundo y este fenómeno se ha convertido en parte de la cultura del bienestar. Tenemos como ejemplo el payaso Chirajito de El Salvador, que le dió una utilidad social al oficio de payaso al reunir a niños y niñas de la calle y enseñarles el oficio en su creación El circo de los niños, así como otras grandes aportaciones a esta modalidad artística circense como el Congreso de payasos, promoviendo los derechos humanos, la creatividad y el respeto mutuo entre comunidades (Melara-Martínez, 2011). Por todo, difícilmente se puede decir que el trabajo del payaso sea simple y fácil.

¿Qué es, por lo tanto, ser payaso? Como define Guarnizo (2013), el payaso puede influir en la sociedad transmitiendo sus valores a través del humor. Asimismo, el payaso también es capaz de enseñar y sensibilizar, y puede transformar los pensamientos de los demás. Transmite y estimula los valores y las emociones que fortalecen las capacidades personales y colectivas (Ros-Clemente y Úcar, 2013). Por lo tanto, el acto del payaso se puede tomar como una disciplina artística que hace posible el autoconocimiento, la cual se basa en el aprendizaje en base al respeto y la aceptación, ya que basándose en una metodología asentada en la risa y el humor, la aceptación de uno mismo lleva a la aceptación del prójimo.

En muchas ocasiones el trabajo del payaso se ha situado dentro del desarrollo del circo, y hoy en día tenemos indicios de ello, ya que el payaso y el circo son vistos como herramientas pedagógicas por los profesionales de la educación y el trabajo social. Tenemos un ejemplo de ello en Cataluña (España) donde la formación artística y social que ofrece la plataforma Ateneu Popular 9 Barris gestionada por la organización Bidó de Nou Barris, tiene como eje central el circo. Esta iniciativa comenzó haciendo diversos talleres de circo y después llevó a cabo acciones con la participación vecinal, convirtiendo el circo en herramienta comunitaria para mejorar el entorno social próximo (Alcántara, Llotje y López, 2009). 
El proyecto Circo en acción (2017), promueve el abrir las puertas a la expresión y a la creatividad, fundamentándose en que éste impulsa la autonomía, la responsabilidad y los valores de la ciudadanía. Este proyecto, a través del arte del circo, cree que los centros educativos pueden convertirse en espacios de juego, encuentro e intercambio de relaciones entre los componentes de la comunidad educativa para promover la solidaridad entre iguales.

Esta modalidad de circo social tiene objetivos similares a los de la nueva modalidad de teatro llamada Teatro del Oprimido, siguiendo la Pedagogía del Oprimido de Freire (1970), basado en su pensamiento y su práxis y también influenciada por la modalidad de Teatro de Brecht. El dramaturgo y educador social Augusto Boal creó el Teatro del Oprimido, como una nueva modalidad que, valiéndose de diferentes técnicas dramáticas, sirve para denunciar las injusticias sociales y las situaciones sociales de miedo, opresión y/o exclusión (Boal, 2011).

Esta novedosa práctica pedagógica llevada a cabo a través del teatro, propone un sistema educativo a favor de los oprimidos. Así, esta técnica artística coincide con la pedagogía de la concienciación de Freire (2005), puesto que al igual que en la Pedagogía del Oprimido todo individuo es protagonista de su educación. En el Teatro del Oprimido, Boal también cree que todos podemos aprender y enseñar, siempre y cuando sea a través de relaciones basadas en la horizontalidad; estando todos a la misma altura, sin oprimidos ni opresores, y respetando al prójimo (Molina-Montecinos, 2005).

En el ámbito escolar también encontramos experiencias sobre el uso del clown como herramienta pedagógica y didáctica en el aula (HobdayKusch \& McVittie, 2002), donde mediante técnicas artísticas se trabajan y profundizan la creación de procesos discursivos del alumnado. En esta misma línea Ruch, Platt y Hoffman (2014) han subrayado las potencialidades de las técnicas del clown en aula de cara a la mejora en los procesos de enseñanza-aprendizaje.

De todo ello se deriva que no es sencillo clasificar a los payasos y que quizá el criterio más habitual es en función de la finalidad de su intervención. De ahí que se diferencien los payasos artísticos/escénicos (Robb, 2007; 
Salcedo-Barzola, Castro-Salcedoy Rivadeneira-González, 2018; Starobinski, 2004), los terapeúticos (Ford, Courtney-Pratt, Tesch \& Johnson, 2013; Galindo-Caicedo y Garzón-Benavides (2015); Kontos, Miller, Mitchell \& Stirling-Twist, 2017; Linge, 2012) y los que intervienen en el ámbito social (Christen, 1998; King, 2017; Shepard, 2011). Es en esta última clasificación donde, a su vez, se distinguen a los payasos comunitarios, humanitarios, rebeldes y socioeducativos (Ros-Clemente y Úcar, 2013). Cada uno de ellos con funciones distintas pero que tienen como denominador común la defensa de los valores universales (Ros-Clemente, 2015b):

- Comunitario: busca la construcción y búsqueda de creación de espacios de juego cómico-poéticos públicos, con el objetivo de llegar a conseguir la implicación de la comunidad.

- Humanitario: promueve ayuda humanitaria a través de formación y/o espectáculos para la mejora de las condiciones de vida en zonas afectadas por desastres naturales, conflictos, etc.

- Rebelde: trata de romper el poder de las jerarquías, poder bélico y militar, con la desobediencia civil y la participación política a través de la risa.

- Socioeducativo: trabaja con procesos socioeducativos favoreciendo el desarrollo de la ciudadanía en todas sus dimensiones: personal, social o comunitaria, y crítica (Gayà-Coll, 2018; SocialClown, 2014).

Por lo que a este último se refiere, al hablar de payasos que trabajan dentro del ámbito de la sociedad y de la educación, se asume también que dentro de ésta la educación especial y la de los adultos, cobran un sentido especial y una atención cuidada. Aunque temas transversales como la educación para la salud y la animación sociocultural son objeto de atención. En definitiva, una modalidad de payaso que atendiendo a cuestiones para las que la sociedad reclama su atención, las aborda como objeto para la mejora de la ciudadanía y el cambio social (Ros-Clemente, 2015b).

Siguiendo a este mismo autor, el payaso socioeducativo hace uso del humor como principal herramienta para la práctica comunitaria y crítica para incidir en esa función socioeducativa. Asimismo, este tipo de

Prospectiva. Revista de Trabajo Social e intervención social No. 27, enero-junio 2019: pp. 159-186 (c) (7) 
payaso, refleja y transmite los valores humanos, sentimientos positivos y emociones democráticas que influyen en las dimensiones personales, sociales y críticas de la animación sociocultural que define Ventosa (2008). En otra clasificación de la modalidad del payaso (circense, promocionador de restaurantes, artístico, teatral, social y hospitalario) que el británico payaso King realizó en una investigación en una estancia en Colombia, también reafirma la labor social del payaso el cual educa a la ciudadanía en el espacio público.

Dicho de otro modo, y como mencionan los autores Ros-Clemente y Úcar (2013) en cuanto a la dimensión personal "se refiere a que la persona adquiere valores de honestidad, aceptación, sinceridad, alegría, entre otros, obteniendo capacidades de disfrute de la vida y cognitivas como imaginar, percibir, razonar" (Ros-Clemente y Úcar, 2013, p. 16). Respecto a la dimensión sociocomunitaria, "existe una transmisión de valores humanos y positivos, que consiguen implicar a la persona en la comunidad a través del arte, dotándola de la capacidad para participar en la vida social y en las relaciones" (Ros-Clemente y Úcar, 2013, p. 16). En cuanto a la dimensión crítica, "posibilita ver el otro lado de las cosas, poniendo en duda las jerarquías y leyes, al tiempo que potencia la capacidad de elección" ( $p$ 16). Pero, además, trabajan por solucionar los problemas y, para ello, se sumergen en el fracaso, conflicto y la debilidad humana (Medina-Arango, Hoyos-Uribe, Bohórquez-Aristizabal y Posada-Vargas, 2015).

\section{La trayectoria de los payasos vascos en las últimas décadas}

A pesar de que el circo y el payaso se crearon hace muchos siglos, los payasos vascos comenzaron su andadura hace pocas décadas. Kixki, Mixki y Kaxkamelon, en el año 1972, fueron los primeros payasos en actuar haciendo uso de la lengua vasca (idioma autóctono del País Vasco y que en adelante nos referiremos a él como euskera) (Ibargutxi, 2014).

En la década de los 70 no había diversión en euskera para los niños y niñas, y con la intención de cubrir ese carencia, comenzaron a trabajar estos primeros payasos. Sus primeros trece años los dedicaron a hacer actuaciones de pueblo en pueblo con la intención de extender el euskera a 
través del humor, y fue en 1982 cuando se convirtieron en Txirri, Mirri y Txiribiton (Murua2, 1990).

Su modalidad de payaso, digamos que era la tradicional: uno (Txirri), payaso cara-blanca serio e inteligente, y los otros (Mirri y Txiribiton) los payasos rebeldes. Estos payasos tuvieron trabajo con el euskera, puesto que, tenían que usar un lenguaje que pudiesen entender todas las personas del País Vasco. Por eso, tuvieron que crear registros diferentes haciendo uso del euskera unificado (dado que esta lengua posee diferentes dialectos), pero sin que quedase un lenguaje frío, para que fuese cercano a los niños y niñas; digamos que más amistoso. Asimismo, hacían uso de un humor fácil para obtener la atención de los niños y niñas, aunque también tocaban otros campos (chistes políticos y de contenido sexual, por ejemplo), y sin hacer uso de un humor demasiado inteligente (Murua2, 1990) sino accesible para todas las personas de cualquier condición social.

Al final de esa década (1979), se creó el grupo de payasos Kakax, Kokox y Kirriki que actuaron durante 20 años, y un año después, se creó el grupo de animación Azkona que comenzó a usar técnicas cirquenses y la expresión corporal con los niños y las niñas. Después de actuar dentro y fuera del País Vasco, en 1988 participaron en el espectáculo del Circo del Mundo y de ahí nació el grupo de payasos llamado Kiki, Koko, Moko y Flash (Ibargutxi, 2014; Argia, 2005).

Comparado con los anteriores, el carácter de los payasos fue cambiando (al payaso Kiki le gustaba jugar, Koko era alegre y Moko el inocente), y también trajo novedades a los payasos vascos que hacía poco acababan de crearse. Como no había ocurrido hasta entonces, comenzaron a utilizar diferentes tipos de técnicas: el monociclo, los malabarismos, las mazas, las pelotas y los diábolos, entre otros (Kiki Koko Moko, 1980).

A la par que estos se crearon en el año 1987, los payasos Takolo, Pirrutx y Porrotx, aunque más tarde Pirrutx dejó el grupo y se unió Pirritx: completando el trío Takolo, Pirritx eta Porrotx (Ibargutxi, 2014).

Estos también comenzaron su andadura como payasos con el fín de promover el euskera acercándolo a los niños y las niñas desde la emoción y la alegría (Ibargutxi, 2014; Argia, 2005; Goikoetxea, 2016; Unanue-Irureta, 2012). Pero esos payasos que se juntaron para sólo un día, siguieron 
de pueblo en pueblo haciendo espectáculos durante muchos años y hoy en día también continúan en ello.

Como ya se ha comentado, Pirrutx dejó el grupo y en 1997 se unió una mujer al mundo de los payasos por primera vez en el País Vasco: Pirritx. Al cabo de unos años, en el 2004, Takolo también tuvo que dejar el grupo, y al poco, en el 2007, se unió la segunda mujer, Marimotots; y hoy en día el grupo de payasos lo constituyen Pirritx, Porrotx y Marimotots. Si bien estas mujeres participaban puntualmente en las actuaciones haciendo de otros personajes, se unieron de manera permanente al mundo del circo y este hecho es importante porque su incorporación era una novedad en una disciplina artística casi exclusivamente reservada a los varones en el País Vasco (Unanue--Irureta, 2012).

En 1996, se creó el trío Gari, Montxo y Joselontxo. Estos, sin embargo, hacían otra modalidad de payaso; la clásica con el añadido de que eran también músicos: un payaso cara-blanca tocaba la trompeta (Gari), y dos augustos (Montxo y Joselontxo) que tocaban el saxo y la tuba (Ibargutxi, 2014). Asimismo, los espectáculos que realizaban eran tanto para niños y niñas como para adultos, y su único objetivo era que el público se lo pasase bien sacándoles una sonrisa (Argia, 2005).

En 1999, Korri, Xalto y Brinko, teniendo el euskera como herramienta principal de trabajo, comenzaron en las fiestas de carnavales de su pueblo -Lezo- (Gipuzkoa, España) con la intención de dar a conocer los lugares, historias y personajes famosos del pueblo, además de trabajar como cuenta cuentos con la intención de promover valores universales (Korri, Xalto ta Brinko, 1999).

Es a partir del año 2000, cuando empiezan a proliferar los grupos de payasos en el País Vasco. En el 2001, siguiendo el estilo clásico, se creó la pareja Poxpolo y Mokolo; al año siguiente, Potxin y Patxin, más cercanos al mundo circense. Y entre los años 2002-2003, siguiendo el recorrido de sus padres, se creó el grupo integrado por Txirri, Mirri y Txiribiton Junior, quienes actuaron junto a los senior hasta que éstos se retiraron en el 2005. 


\section{Objetivos de investigación}

La literatura científica nos recuerda que es necesario realizar investigaciones sobre los payasos en general y respecto a la modalidad de payasos educativos en particular: "La investigación científica, la formación y la medición del impacto referente a clown socioeducativo son inexistentes" (Ros-Clemente, 2015b, p 2). Es por ello que la pregunta de indagación que ha movilizado esta investigación sea ¿Cómo se crea un modelo educativo desde un grupo de payasos circunscritos en el país Vasco? Para poder dar respuesta a esta pregunta, se ha analizado el origen y el desarrollo del proyecto pedagógico de los payasos Takolo, Pirritx, Porrotx y Marimotots, puesto que su actividad es conocida, reconocida y difundida de forma activa por todo el País Vasco.

Los objetivos de investigación son los siguientes:1. Identificar las características del supuesto modelo educativo; 2. Comprobar si el grupo de payasos, objeto de estudio, ha creado un nuevo modelo pedagógico; 3. Interpretar las aportaciones que el supuesto modelo pedagógico hace al mundo educativo.

\section{Metodología}

La investigación se ha enfocado desde el paradigma cualitativo e interpretativo, dado que es idóneo para analizar pensamientos, reflexiones y actitudes, al intentar reunir las vivencias de las personas investigadas para que quien investiga comprenda sus puntos de vista (Janesick, 2000). Asimismo, en tanto que la investigación social se encuentra dentro del paradigma interpretativo, puesto que el objeto de estudio - la modalidad de payasos socioeducativa- es consecuencia de una situación histórica y del valor cultural que tiene en la educación de niños y niñas, en nuestro caso, del País Vasco (Blaxter, Mughes y Tight, 2008; Martínez-Rodríguez, 2011). Huelga decir que la cultura vasca y la situación del País Vasco intervienen directamente en la explicación de la creación y el desarrollo de este fenómeno social. 
En sintonía con lo dicho anteriormente, el método de investigación ha sido el Estudio de caso. Esta estrategia metodológica nos ha permitido aproximarnos a la comprensión de la evolución y quehacer del grupo de payasos del País Vasco Takolo, Pirritx, Porrotx y Marimotots como caso (Bodgan y Biklen, 1982; Stake, 1998; Vázquez-Recio y Angulo-Rasco, 2003).

Para poder llevar a cabo esta investigación, y entender las razones de los cambios realizados desde sus orígenes hasta hoy en día, se ha hecho uso de ciertas herramientas y técnicas de investigación que han sido:

Entrevistas individuales en profundidad a cada uno de los cuatro payasos. Como señalan Jordi-Sánchez y Macías-Gómez (2014) se ha tenido presente que al entrevistar, el saber escuchar es tanto o más importante que el saber preguntar. Partiendo de este supuesto, la entrevista en profundidad ha facilitado: una implicación interpersonal con el entrevistado, una aclaración de cuestiones o dudas que han surgido a lo largo de la entrevista, y una observación gestual de lo que acontecía (información de carácter subjetivo y de tipo biográfico) que se ha ido registrando rigurosamente (Lukas y Santiago, 2009).

Grupo de discusión con los cuatro payasos. Esta técnica de carácter flexible y abierto ha promovido la participación e interacción grupal, la construcción de la información en el propio escenario grupal donde los payasos, estimulados por la presencia del resto del equipo, han emitido sus opiniones obteniendo, así, información de primera mano (Callejo, 2001; Infesta, Vicente y Cohen, 2012; Morgan y Krueger, 1993).

Análisis documental de dos entrevistas televisivas en torno a su modalidad de payaso y proyecto, y el análisis de soportes tales como: espectáculos, $D V D$ s, vídeos y cuentos, y documentos creados por el grupo de payasos.

En cuanto al procedimiento analítico, se han seguido las pautas planteadas por Miles y Huberman (1994, cit. en Rodríguez-Gómez, GilFlores y García-Jiménez, 1996). Dicho proceso consiste en ir diferenciando unidades de significado a los que se les ha asignado una categoría, que se ha ido concretando según su contenido. Las categorías se refieren a opiniones, actitudes, sentimientos, valoraciones, etc. que han expresado 
los participantes en la línea que apuntan (Gil y Perrera, 2001 citado en Lukas y Santiago, 2009).

La construcción del sistema categorial ha sido emergente (ver Tabla 1). No obstante, debemos tener en cuenta que en las entrevistas en profundidad se han planteado preguntas tras una primera revisión bibliográfica. Es importante señalar al respecto, que estas preguntas han sido acomodadas a la información recogida posibilitando el surgimiento de nuevos temas. Las categorías surgen del análisis de esta información, lo que se puede considerar un método mixto, teniendo en cuenta el carácter flexible y abierto que caracteriza a este estudio, en la línea que apunta (Cabrera 2000, citado en Lukas y Santiago, 2009).

Tabla 1. Sistema categorial

\begin{tabular}{|c|c|c|}
\hline \multirow{5}{*}{ Comienzo } & \multicolumn{2}{|l|}{ Origen } \\
\hline & \multicolumn{2}{|l|}{ Tipo de payaso } \\
\hline & \multicolumn{2}{|l|}{ Motivos para ser payaso } \\
\hline & \multicolumn{2}{|l|}{ Referentes } \\
\hline & \multicolumn{2}{|l|}{ Objetivo inicial } \\
\hline \multirow{5}{*}{ Desarrollo } & \multirow[b]{2}{*}{$\begin{array}{l}\text { Origen del proyecto } \\
\text { pedagógico }\end{array}$} & Modo y razón de la creación \\
\hline & & $\begin{array}{l}\text { Normalización del euskera y } \\
\text { desarrollo comunitario }\end{array}$ \\
\hline & \multicolumn{2}{|l|}{ Objetivo actual } \\
\hline & \multicolumn{2}{|l|}{$\begin{array}{l}\text { Claves del payaso e ideas a } \\
\text { transmitir }\end{array}$} \\
\hline & \multicolumn{2}{|l|}{ Determinante sen la evolución } \\
\hline $\begin{array}{l}\text { Aportaciones a la } \\
\text { educación }\end{array}$ & Proyectos y material didáctico & \\
\hline
\end{tabular}

Fuente: elaboración propia

De este modo, el Comienzo, el Desarrollo y las Aportaciones a la educación han sido las metacategorías. En la primera, el Comienzo, se encuentran las categorías de: el origen del grupo de payasos, el tipo de payaso, la razón de elegir ser payaso, los referentes y el objetivo principal. En la segunda, Desarrollo, con el fin de explicar el origen de la creación del proyecto pedagógico, se encuentran dos subcategorías: el modo y la razón de la creación, y la normalización del euskera y el desarrollo comunitario. Otras categorías serían el objetivo actual; las claves del payaso y las ideas 
que quieren transmitir, así como los determinantes en su evolución. En la tercera y última metacategoría, las Aportaciones a la educación, se encuentran los hallazgos en torno a los proyectos y el material didáctico. Ha sido el sistema categorial el que ha facilitado el análisis y la interpretación de los datos.

En cuanto a los factores éticos y de calidad subrayemos que, en sintonía con lo que proponen Blaxter, Hughes y Tight (2008) respecto a los participantes, se les dejó claro desde el principio la naturaleza del estudio, se les explicó en qué consistía el consentimiento informado, y se llegó a acuerdos sobre el uso de los datos, su análisis y divulgación. Además, se han codificado las voces de los y las payasos (como puede verse en las voces recogidas en el apartado de resultados), y ello es un elemento más para preservar la privacidad y el anonimato, y valores como la dignidad e integridad. Así mismo, el respeto por su libertad y autonomía se han defendido en todo momento tal y como indica el Comité de Ética para las investigaciones relacionadas con Seres Humanos (CEISH).

Respecto a la calidad de la información, y siguiendo a Tójar-Hurtado (2001), el criterio por excelencia para la investigación naturalista es la veracidad que se articula y se plasma en este trabajo a través de las siguientes características: a) Credibilidad: se ha intentado recopilar toda la información posible con la pretensión de reconstruir el modelo de intervención socioeducativo de este grupo de payasos (Tójar-Hurtado, 2001). En este sentido y siguiendo a Lincoln y Guba, 1985 (citado en Rodríguez-Gómez, Gil-Flores y García-Jiménez, 1996), para subrayar la credibilidad se han puesto en práctica estrategias de triangulación y la comprobación con los y las participantes. En esta línea, se ha dotado de veracidad a través del uso de la triangulación de fuentes (payasos y, documentos y materiales) permitiendo de esta forma contrastar los datos e interpretaciones; b) Transferibilidad: Se pretende transferir lo realizado en este estudio a otros contextos similares al analizado y, en cierto sentido, la mayoría de los grupos de payasos y sus actuaciones pueden ser objeto de transferencia; c) Dependencia: En este estudio se han aplicado diferentes técnicas (grupo de discusión, entrevistas, análisis documental) que han permitido obtener resultados muy similares, lo que permite deducir el 
carácter de estabilidad de los mismos. Finalmente, d) Confirmabilidad: se subraya al utilizar descriptores de bajo nivel de inferencia (las transcripciones) registrando lo más fielmente posible la realidad.

\section{Resultados e interpretación}

En toda la trayectoria de los payasos vascos no hay ningún otro payaso que haya creado un modelo pedagógico que tenga características de esta índole, es decir, el grupo de payasos investigado ha creado un nuevo modelo pedagógico; entre otros porque proyectan muchas de las características de la sociedad vasca; impulsan el euskera y los valores asociados a las minorias étnicas; abordan temas cotidianos; trabajan en colaboración con diversas asociaciones; fomentan la alegría y un punto de vista especial sobre el humor ... y es un modelo en constante proceso de cambio y reflexión como se recoge en la siguiente voz:

Los payasos (...) hemos tenido un recorrido. Comenzamos en Lasarte-Oria fomentando el euskera en la comunidad. El euskera y la alegría, y después los valores. Y hemos ido aprendiendo durante el recorrido, hemos ido expandiendo, hemos expandido un poco el País Vasco. Hemos trabajado diferentes soportes. Y también nos sentimos parte de la concienciación del euskera en nuestro pueblo, seguimos en Lasarte-Oria, seguimos en la fiesta del Olentzero, 35 años después, y seguimos participando en sus proyectos (...). Tenemos un recorrido paralelo (...), pero conjuntamente, el recorrido de la concienciación del euskera, el de nuestro grupo, cómo vemos nuestro pueblo, cómo vemos el País Vasco (E1b, 39:23).

La cultura y la sociedad vasca son el centro de su actividad. Comenzaron como payasos con la intención de acercar a aquellos niños y niñas al euskera, con la creencia de que tendría que ser desde la alegría y el humor. Para ello, tuvieron como referencia esquemas de otros payasos clásicos y apropiándose de las ideas provinientes de las experiencias de otros. Por ello, la evolución del grupo de payasos le debe mucho al euskera, de forma que hoy en día se siguen situando dentro de la actividad cultural vasca: 
Para nosotros era importante transmitir el euskera de forma alegre, proque en los centros educativos era una asignatura, en Lasarte-Oria el $85 \%$ era alumnado del modelo $\mathrm{A}$, y el euskera para los niños y niñas era una asignatura. (...) Entonces nosotros pensamos que para transmitir el euskera había que hacerlo desde la alegría, de una forma divertida y a nosotros nos paració que hacer de payaso era (...) una manera para ello. Y (...) empezamos por eso (E1b, 45:14).

O como comenta otro de los participantes: "Le debemos mucho al euskera, le debemos: nuestra forma de vida, nuestra perspectiva, nuestro oficio, todas nuestras relaciones..." (E1a, 1:54:58).

Los objetivos principales que pretenden lograr son la normalización del euskera, transmitir alegría como vehiculizadora de valores educativos. Manteniendo la perspectiva de los objetivos del principio, hoy en día continúan fomentando a través de la disciplína artística del payaso la perspectiva educadora de la alegría y el euskera. Asimismo, el haber dado el salto al modelo pedagógico les creó la necesidad de sumar un tercer objetivo: los valores. Esto fue debido a que comenzaron a trabajar temas de calado social.

Trabajamos temas como esos, (...) los que vivimos de cerca, desde la comunidad, los que recibimos de los medios de comuninación, los que nos llegan, los temas que nos aportan, temas que nos aportan y que pueden aportar, los que podamos fortalecer transmitiéndolos a la sociedad (E1a, $1: 55: 32)$.

Una vez metidos en la dinámica de elaborar temas sociales, y si entendemos que la modalidad de payaso que comenzaron a realizar era basada en los acontecimientos de la sociedad, el trabajar en valores era imprescindible como herramienta pedagógica en su modelo educativo. En definitiva, reconocen trabajar en valores porque se sienten educadores y educadoras y que para ello se valen de su influencia en tanto que ellos tienen un impacto directo e inmediato en los menores y su entorno.

Ha sido determinante el empezar a colaborar con diversas asociaciones. Del mismo modo que reivindican que el euskera, el País Vasco y la cultura vasca deben tener su sitio en el mundo, también creen que ese mundo debe 
ser para todas y todos. En ese sentido, tiene vital importancia el colaborar con diversas asociaciones del País Vasco, y el hecho de que hoy en día participen también en el proyecto Bizipoza, les sirve para reivindicar un mundo y un País Vasco que sea para todas y todos desde la perspectiva de los débiles y los excluídos. De este modo, a través de los payasos, se divulgan en euskera los mensajes y los valores de estas asociaciones:

Para nosotros ese trabajo realizado con las asociaciones ha sido determinante. (...) Porque nos han dado para nuestro oficio, pero para el humor, pero también para nuestras vidas: una mirada, una perspectiva, sobre el mundo, y desde el País Vasco (E1a, 1:50:31).

(...) Nos parece, (...) que el País Vasco es un pueblo, donde la gente tiene una disposición para: donar sangre, transplantar órganos, trabajar de forma colaborativa, participar en iniciativas de forma activa, ayudar. Y nosotros lo que queremos es lograr una amplia red de voluntariado en torno a estos temas sociales y a niños y niñas con necesidades especiales, sobre las asociaciones de estos niños y niñas, que funcione en euskera. Y que vaya a fortalecer todo esto y que socialice estos valores, que ayude en todo ello (E1b, 5:44).

A medida que ven que el trabajo del payaso puede ser transformador, el comenzar a trabajar con asociaciones ha sido un elemente determinante y aglutinante para el grupo y para la evolución del modelo pedagógico, ya que a través de esta nueva modalidad del payaso, partiendo del euskera y la alegría, han podido difundir el trabajo de estas asociaciones y los diferentes temas sociales que abordan. Por consiguiente, con el fin de satisfacer los derechos de las personas, y trabajar valores transformadores desde una perspectiva inclusiva, ha sido fundamental la colaboración con otras asociaciones y entidades sociales.

El desarrollo del modelo educativo todavía es un contínuo proceso de cambio. El proyecto educativo que este grupo de payasos sustenta, que si bien está alimentado por la sociedad vasca y el euskera, es en gran medida autodidacta: creaban por su cuenta los espectáculos o iban haciendo cambios según iban probando cosas. De este modo, dejaron a un lado los sketch y comenzaron a elaborar historias, y todo lo que hacían tenía un porqué y un sentido educativo.

Prospectiva. ReVista de Trabajo Social e intervención social No. 27, enero-junio 2019: pp. 159-186 Cc) (i) () () 
La evolución de estos payasos es consecuencia de un proceso de cambio contínuo auspiciado por el momento y la situación histórica, y es por ello que aún perduran en ese interminable proceso de búsqueda y tansformación al son de los cambios de la propia sociedad. Por lo tanto, el proceso de completar el proyecto pedagógico es una tarea siempre en estado inconclusa. Es por el hecho de que la sociedad esté cambiando contínuamente que los payasos tengan que estar atentos a los cambios en los temas cotidianos, y responder haciendo uso de la creativad a las nuevas necesidades emergentes.

La razón de crear un modelo educativo, es consecuencia del contínuo proceso de reflexión. Concluyendo que la sociedad y la educación están cambiando contínuamente, creían que su modalidad de payaso tenía que ser el reflejo de dichos cambios, y por ello apostaron por un modelo educativo, porque creían que era necesaria dicha evolución. Es decir, opinaban que su trabajo como payasos tenía también que intentar dar respuesta a esos cambios, amoldándose a la sociedad:

Los modelos que se están planteando ahora en las escuelas, los cambios de modelos escolares, espacios más amplios, para que pierdan un poco ese formato de fábrica, dar confianza, cercanía, fuera de rigurosidad, fuera de plastazos y patadas, y de la violencia hacia los menores y familias... todo ello tambíen tiene que verse reflejado en los payasos, si no estaríamos aburridos hace tiempo (E3, 32:49).

De ese modo, el modelo educativo que crea el grupo es consecuencia de estar sometidos a un contínuo proceso de reflexión. Por lo tanto, esta modalidad de payasos aúne la reflexión y la acción, es decir, las contínuas reflexiones marcan su dirección, y la práctica les sirve para afinar la reflexión y marcar su rumbo.

El ser vasco, ser pequeño, tiene mucha relevancia en este modalidad de payaso. Al igual que el grupo, en un principio se creó de la necesidad de la normalización del euskera, la modalidad de payasos que trabaja este modelo pedagógico se creó para dar respuesta a otras necesidades del País Vasco. Asimismo, no se puede entender la evolución de este grupo de payasos sin tener en cuenta el contexto Vasco, puesto que se alimenta 
del intercambio con diversos agentes. Por lo tanto, la situación del País Vasco y el hecho de ser vascos tienen importancia si queremos entender la evolución que ha tenido esta modalidad de payasos:

Cuando eres pequeño, y cuando tienes una lengua minorizada, como los salmones, saltando, a contracorriente, tienes que luchar, tienes que ir hacia adelante. Y cuando eres pequeño, y cuando tienes que luchar por el pueblo para seguir adelante, puedes crear una empatía hacia los que son pequeños como tú, hacia esas reivindicaciones. Puedes lograr empatía: hacia las mujeres, con una perspectiva de género; hacia las esas relaciones que serán entre todas y todos, con una perspectiva hacia la igualdad; o hacia un modelo económico; o un modelo de justicia; o hacia la ecología; o hacia las relaciones del mundo. Entonces, de alguna manera, el ser pequeño te puede dar (...) esa mirada, esa empatía y entonces, la propia lengua, el euskera, no es para nosotros sólo una herramienta para comunicarnos, es un medio (...) para transmitir los valores (E1a, 1:50:57).

Podemos meter la pata, pero la sacamos rápido; nos conocemos entre todas $\mathrm{y}$ todos, trabajamos en red, trabajamos colaborativamente... El ser pequeño también da ventaja y nuestro pueblo también tiene eso (E1a, 1:53:51).

De esta manera, creen que el idioma y la cultura son transformadoras, $y$, teniendo en cuenta que su modalidad de payasos es tan influenciada por ambas, no es casualidad que esta nueva modalidad esté hecha desde una perspectiva pedagógica y transformadora; al igual que no es casualidad que se encuentren situados dentro de la sociedad vasca. Por todo ello, el ser pequeños para ellos se ha convertido en un valor añadido de suma importancia, ya que eso les permite tener muchas ventajas a la hora de crear su proyecto educativo: tener la oportunidad de juntarse con diversas asociaciones, poder llevar a cabo proyectos con agentes sociales y educativos, tener cercanía con todos los que colaboran, poder transmitir valores...

Nos parece que los vascos y vascas podemos tener un papel especial en todo ello, que tenemos una ventaja. A mí no me parece que sea casualidad, me parece que el euskera también, el ser vasco o vasca nos da esa mirada, que el ser pequeño nos ha dado eso (E1a, 1:50:48). 
Con esa humildad de ser pequeños de los vascos, han practicado la empatía, ya que partiendo de las desventajas de uno mismo se entienden mejor las desventajas del prójimo. La colaboración que realizan con diferentes agentes sociales y educativos gracias a esa empatía es gracias a la sociedad vasca, característica propia de los vascos, y la de la colaboración.

Todos los soportes que crean están realizados desde una mirada pedagógica. Metidos en la dinámica del desarrollo de un modelo pedagógico, el grupo de payasos tuvo que hacer una reflexión sobre la globalización de hoy en día. De este modo, pensaron qué espacios se le ofrecía al euskera dentro de este mundo globalizado. Por consiguiente, viendo que en los materiales y soportes que se encuentran dentro del mundo infantil (juegos, ropas, materiales escolares o dibujos animados, entre otros) predominan otros idiomas, reflexionaron en torno a que las niñas y los niños necesitaban recursos en euskera. Por ello, pensaron en ofrecer materiales y soportes en euskera y, tal como lo pensaron, fueron capaces de crearlos.

A raíz de esto, crearon también un club y una revista: Irrien Lagunak $K l u b a$, y la Revista Irria. Trabajando a través de ellos valores en torno a la salud, naturaleza, ciencia, cultura y solidaridad con los niños y las niñas, promoviendo una intervención educativa en euskera.

Además de eso, pensaron que también se debían ofrecer otros recursos, siempre y cuando estos productos se crearan desde una perspectiva pedagógica y estuvieran relacionados con la cultura vasca, el euskera y las emociones. De ese modo, en lugar de tomar estos productos como merchandising, los asumieron como productos culturales y didácticos, en tanto que su objetivo no era lucrarse económicamente con ellos o aumentar su fama, sino poder educar en euskera.

Y después, pues sí, también hacemos cosas que (...) sirven para el formal. (...) No hacemos algo, aunque también sea didáctico, por hacer. Siempre tiene... normalmente tiene siempre algo atribuído. Intentamos darle un valor añadido (E3, 40:40).

Por lo tanto, tiene un porqué pedagógico: crean diversos soportes y recursos para poder educar a las niñas y niños, los cuales pueden ser útiles 
en todos los ámbitos educativos y sirven para difundir mensajes latentes en la sociedad actual.

La clave es participar en proyectos con otros agentes. Para trabajar temas sociales ha sido clave, según los payasos, el contacto y el trabajo colaborativo con asociaciones y diferentes agentes sociales y educativos. De ahí que hayan participado en proyectos, como por ejemplo: Irrien Lagunak Kluba, Bizipoza, Oinherri y Lur eta Amets.

El proyecto social Bizipoza es un proyecto que funciona en euskera, y los payasos pretenden ayudar a difundir en la sociedad los valores que transmiten las asociaciones que se encuentran dentro de él. Por otro lado, también están implicados en el proyecto Oinherri, que desde la comunidad, tienen como objetivo el fomentar la verdadera participación de los niños y las niñas y difundir valores educativos. Además, participa la cooperativa de los payasos en el proyecto Lur eta Amets, el cual tiene como finalidad difundir la historia del euskera y del País Vasco. Por lo tanto, la Cooperativa Katxiporreta tiene su propia labor como grupo de payasos, pero también participa en colaboración con otros agentes en otros proyectos. Estos proyectos realizados desde la colaboración tienen como propósito: hacer defensa de los derechos sociales; que la responsabilidad de la educación de las niñas y los niños sea de toda la sociedad; que la educación sea participativa y comunitaria; y reivindicar que el País Vasco y el euskera tengan su propio lugar en el mundo. Para ello, estos payasos que son referentes simbólicos y sociales tienen un quehacer importante difundiendo a través de la disciplína artística de los payasos las ideas que se engloban en los proyectos en que participan.

Gracias a esta modalidad educativa de payaso han hecho muchas aportaciones a la educación. Durante toda la historia del grupo, los payasos han transmitido valores y trabajado temas sociales que han sido proyectados en espectáculos, y éstos a su vez grabados en DVD. Junto a ello, las canciones que acompañan las actuaciones también han sido objeto de publicaciones de discos y eso ha dado pie a que se puedan trabajar los valores y temas sociales en el ámbito del hogar y en los centros educativos.

Mayormente, las aportaciones que se han hecho al mundo de la educación han sido creadas por las peticiones de la gente; proyectos creados 
a partir de las necesidades que se iban identificando. El proyecto familiak milakolore es ejemplo de ello. El hecho de que faltaran materiales para explicar la multitud de composiciones familiares en la sociedad del siglo XXI, dio lugar al juego de cartas que se creó para mostrar los diferentes modelos familiares.

Las aportaciones que han hecho estos payasos han sido útiles no sólo en la educación formal, sino también en la no-formal y en la informal, puesto que los productos que han creado ofrecen la oportunidad para trabajar los valores universales y los temas sociales de actualidad más allá de los muros de clase y de los hogares, llegando a los barrios y sus calles.

Una forma de educar fuera de la escuela también. De una forma muy simple explicamos varios temas, (...) y de una forma que ellos y ellas también puedan entender, para que luego también debatan en sus casas. Ciertamente, hacemos para ese no-formal, pero es verdad que en el formal también utilizan nuestras canciones y nuestros vídeos y nuestros reportajes. Nosotros somos conscientes y no lo hacemos un poco mirando a todo ello, pero sabemos que los usan. Nos encontramos con muchos profesores y profesoras y nos dicen y nos dan las gracias por los materiales (E4, 29:02).

En resumen, al igual que ven que el mundo artístico y en concreto el trabajo de los payasos es tranformador, como lo son también la educación y la cultura; creen que puede ser útil para reivindicar un mundo mejor, una sociedad más inclusiva y solidaria. Esa reivindicación la realizan siempre desde una perspectiva comunitaria, desde la equidad, con la intención de obtener una sociedad y una educación democráticas, la cual se construye a través de las relaciones horizontales que plantea Freire (2005); en definitiva, partiendo de las necesidades de los oprimidos.

\section{Conclusiones}

El grupo de payasos Pirritx, Porrotx y Marimotots ha sido pionero en crear un modelo pedagógico vasco dentro de la disciplína artística de los payasos. Es más, sin tener ningún referente en el País Vasco y tampoco en el extranjero, han sido capaces de construir un modelo de payasos propio. Sin embargo, aunque el grupo tiene similitudes (puesto que hacen visitas a 
hospitales o a países no desarrollados, por ejemplo) con los tipos de payasos que explican los autores Ros-Clemente y Úcar (2013), el mayor parecido lo tienen con el payaso social, el cual se encontraría dentro de la modalidad del payaso socioeducativo. Por lo tanto, es el primer grupo de payasos vasco que cumple con las características del payaso socioeducativo que explican los autores Ros-Clemente y Úcar (2013), además de disfrutar de características propias que provienen del contexto específico de actuación: la sociedad vasca. Esto quiere decir que estos payasos, al igual que el payaso socioeducativo, desarrollan lo que se denomina democracia cultural comunitaria.

La novedosa modalidad de payaso socioeducativo, tiene como finalidad el mejorar la comunidad y promover el cambio social a través del payaso. Por lo tanto, este modelo pedagógico creado a través de payasos vascos es muy apropiada con el fin de promover una sociedad inclusiva basada en la democracia. Para poder obtener esa sociedad, es imprescindible dar opción a la comunidad de concienciar y enseñar una perspectiva crítica en todos los ámbitos de la educación, como señalan Hobday-Kusch \& McVittie (2002) y Ruch, Platt y Hofmann (2014) desde la perspectiva escolar.

Este grupo de payasos ofrece una amplia oferta para promover la reflexión desde la infancia, puesto que esa perspectiva educativa les obliga a estar en continuo cambio, amoldándose a la sociedad del momento; y a medida que son agentes sociales, ofrecer esa perspectiva pedagógica a quienes siguen su recorrido como payasos.

Asimismo, la creación de este modelo educativo es consecuencia de haber estado implicados en la sociedad y de estar en continua relación con creadores vascos, puesto que el haber estado en contacto con agentes sociales y educativos les ha proporcionado detectar las necesidades de la realidad, haciéndoles reflexionar en torno a la necesidad de crear un modelo pedagógico para así poder satisfacer esas necesidades.

Esas distintas reflexiones marcan precisamente el rumbo de esta nueva modalidad de payaso. Desde el momento en que los payasos se vieron como agentes sociales, han tenido claro que además de promover sus objetivos inciales como el fomento del euskera y de la alegría, también tenían que trabajar temas sociales y valores universales. A causa de eso, han estado 
atentos a las cuestiones de actualidad ya que a medida que la sociedad va cambiando contínuamente, su modalidad de payaso se va adaptando a ella. Por ello, necesitan una perpectiva transformadora, que provoque en ellos el sometimiento a un continuo proceso de formación y cambio.

Podemos decir también que son payasos investigadores, ya que para poder trabajar con temas importantes para el pueblo, tienen en cuenta los acontecimientos de la sociedad que viven de cerca, haciendo reflexión sobre ellos. Ese proceso en su modelo pedagógico, tiene dos dinámicas: la reflexión y la acción, las cuales se retroalimentan. Sus contínuas reflexiones marcan su dirección, puesto que su práctica está dirigida por las reflexiones. Por consiguiente, se acercan a la praxis de la Investigación-Acción, ya que también podemos considerarlos como payasos investigadores. Esto coincide con lo que dicen autores como Boal (2011), en tanto que las técnicas artísticas pueden servir para denunciar las injusticias y las difíciles situaciones, teniendo en cuenta el contexto socio-histórico del momento.

Por consiguiente, para poder reivindicar a favor de un mundo mejor y de un País Vasco en construcción, deciden qué tema trabajar a través de un proceso de discusión, difundiendo una ideología específica que aúna diversos valores: respeto por la identidad sexual, cultural, religiosa... de cada cual; actitud positiva ante la vida; enfrentarse a las injusticias en colaboración con otras personas y promover un mundo para todas y todos a través de la solidaridad; consciencia en el hecho de que todas y todos tenemos habilidades y carencias, y que con todas ellas se crea la alegría de vivir, y trabajo desde las necesidades, pero sobre todo desde la perspectiva de los más débiles.

Para reivindicar un mundo para todas y todos, es indispensable para los payasos el colaborar con asociaciones de diferente índole, al igual que con los que están relacionados con la cultura vasca o el mundo de la educación. De ese modo, las características de la sociedad vasca tienen relevancia en los procesos educativos, puesto que en el modelo propuesto por Freire (2005), la educación popular sirve para movilizar al pueblo. Por consiguiente, tiene grandes similitudes con la Pedagogía del Oprimido de Freire (2005) y con la metodología del Teatro del Oprimido de Boal (2011), ya que la propuesta de estos payasos también es un modelo pedagógico 
novedoso, la cual a través de los payasos se conciencia a niños y niñas y familias en torno a temas actuales sujetos a crítica, apostando siempre por una sociedad democrática en la que no haya oprimidos.

A raíz de ello, el ser vasco, el ser pequeño, tiene mucho que ver en la creación de esta modalidad de payaso, puesto que el ser pequeño o estar oprimido crea la necesidad de impulsar acciones nuevas; el hacer frente a las necesidades de forma diferente. Asimismo, en la educación se da la horizontalidad de las relaciones humanas cuando se trabaja desde la estructura de los oprimidos y no de los opresores.

En consecuencia, podemos decir que a través del payaso se empodera a la sociedad vasca, ya que conciencian al pueblo gracias a su práctica reflexiva que inducen a su vez a la reflexión del espectador respecto a un mundo más justo y equilibrado.

No queremos concluir sin hacer mención a que el investigar en este campo artístico abre posibilidades de analisis en relación al impacto que proyectos nacidos desde la inquietud de la comunidad, son actuaciones que conllevan una intervención social y educativa que tienden a movilizar al pueblo para mantener e impulsar su lengua, cultura y tradiciones desde una mirada inclusiva. Desde el trabajo y la educación social esta realidad no se puede obviar, sino impulsarla e introducirla en los diseños y propuestas de intervención comunitaria.

\section{Referencias bibliográficas}

Alcántara, A., Llotje, T. y López, J. (2009). El circo social una herramienta educativa y transformadora. Barcelona: Bido Nou Barris.

Argia (2005). Sudur gorrien tribua. Argia. Recuperado de http://www.argia.eus/ argia-astekaria/2014/sudur-gorrien-tribua.

Blaxter, L., Mughes, C. y Tight, M. (2008). ¿Cómo se investiga?, Barcelona: Grao. Boal, A. (2011). Teatro del oprimido. Cuadernos de pedagogía, 411, 46-51.

Bodgan, R.C. \& Biklen, S.K. (1982). Qualitative research for education: an introduction to theory and methods. London: Allyn and Bacon.

Callejo, J. (2001). El grupo de discusión: introducción a una práctica de investigación, Barcelona: Ariel.

Ceballos, E. (1999). El libro de oro de los payasos. Los más famosos y divertidos sketches de circo. México: Col. Escenología. 
Circo en acción. (2017). El Circo hace escuela. Recuperado de http://www. circoenaccion.com/escuela/.

Christen, K. (1998). Clowns and tricksters. An encyclopedia of culture and tradition. Denver: ABC-CLIO.

Crowther, C. (1979). Payasos y payasadas. Bogotá: Voluntad Editores Ltda.

Domínguez, A. (2015). La vulnerabilidad tras la risa. Archipiélago. Revista cultural de nuestra América, 22(87), 38-39.

Fo, D. (2008). Manual mínimo del actor. México: Ediciones el Milabro-UANL.

Ford, K., Courtney-Pratt, H., Tesch, L. \& Johnson, C. (2013). More than just clownsClown Doctor rounds and their impact for children, families and staff. Journal of Child Health Care, 18(3), 286-296. doi: 10.1177/1367493513490447.

Freire, P. (1970). Pedagogía del Oprimido. Montevideo: Tierra Nueva.

Freire, P. (2005). Pedagogía del oprimido. (2 ed.), México: Siglo Veintiuno Editores.

Galindo-Caicedo, C. y Garzón-Benavides, C. (2015). Construcción del rol del doctor clown con énfasis en música en contextos hospitalarios en la Fundación Doctora Clown, Bogotá-Colombia. (Tesis de especialista). Universidad Distral Francisco José de Caldas. Facultad de Ciencias y Educación. Especialización en Desarrollo Humano con énfasis en Procesos Afectivos y Creatividad. Bogotá, Colombia.

Gayà-Coll, M. (2018). El clown i l'humor a la intervenció socioeducativa, (Trabajo de pregrado). Universitat de les Illes Balears. Facultad de Educación. Educación Social. Palma de Mallorca, España

Goikoetxea, G. (2016). Ereindakoaren fruituak ontzen hasteko garaia. Berria. Recuperado de http://www.berria.eus/paperekoa/1924/004/001/2016-08-04/ ereindakoaren fruituak ontzen hasteko garaia.htm.

Guarnizo, M. (2013). El payaso: recreación y educación en una sola experiencia. Bogotá: Universidad Pedagógica Nacional.

Hobday-Kusch, J. \& McVittie, J. (2002). Just clowning around: classroom perspectives on chlidren's humor. Canadian Journal of Education, 27(2-3), 195-210.

Ibargutxi, F. (2014). Pailazo guztiak Mokolo gogoan. Diario Vasco. Recuperado de http://www.diariovasco.com/v/20100417/cultura/pailazo-guztiak-mokologogoan-20100417.html.

Infesta, G., Vicente, A. y Cohen, A. (2012). Reflexiones en torno al trabajo con grupos de discusión en Ciencias sociales, Intersticio. Revista Sociológica de Pensamiento Crítico, 6(1), 233-244.

Janesick, V. (2000). La danza del diseño de la investigación cualitativa: metáfora, metodolatría y significado. En C.A. Denman y J.A. Haro (Comps.) Por los 
rincones. Antología de métodos cualitativos en la investigación social. México: El Colegio de Sonora.

Jara, J. (2011). Los juegos teatrales del clown: navegante de las emociones. Buenos Aires: Ediciones Novedades Educativas.

Jordi-Sánchez, M. y Macías-Gómez, B. (2014). La entrevista en profundidad como recurso pedagógico en los estudios de Trabajo Social y Educación Social. Potencialidades y retos para el aprendizaje teórico-práctico. Revista de Investigación en Educación, 12(1), 105-111.

Kiki Koko Moko. (1980). Kiko, Moko eta Koko pailazoak. Recuperado de http:// www.kikikokomoko.com/.

King, B. (2017). Clowning as social performance in Colombia: ridicule and resistance. London: Bloomsbury.

Kontos,P., Miller,K.L., Mitchell,G.J.\& Stirling-Twist,J.(2017).Presenceredefined: The reciprocal nature of engagement between elder-clowns and persons with dementia. Dementia, 16(1), 46-66. doi: 10.1177/1471301215580895. Gail Joyce Mitchell

Korri, Xalto ta Brinko (1999). Korri, Xalto ta Brinko pailazoak. Recuperado de http://www.korrixaltotabrinko.com/.

Linge, L. (2012). Magical attachment: Children in magical relations with hospital clowns. International Journal of Qualitative Studies on Health and Well-being, 7(1), doi: 10.3402/qhw.v7i0.11862.

Lukas, J.F. y Santiago, K. (2009). Evaluación educativa. Madrid: Alianza Editorial. Medina-Arango, D.M., Hoyos-Uribe, J.M., Bohórquez-Aristizabal, L.F., y Posada-Vargas, R.A. (2015). El clown una herramienta pedagógica. Un acercamiento a las prácticas de resiliencia desde los procesos de creación teatral de los jóvenes de la Corporación Polichinela (Tesis de pregrado). Medellín: Universidad de Antioquia, Facultad de Arte. Depatamento de teatro. Medellin, Colombia.

Rico-Medina, Z.C. (2015). Hacia una definición del payaso. (Tesis de maestría). México: Universidad Autónoma de Querétaro. Facultad de bellas artes. Maestría en Artes con línea terminal en Arte Contemporáneo y Sociedad. Santiago de Querétaro. México.

Melara-Martínez, M.A. (2011). Arte popular, culturas híbridas y patrimonio inmaterial en El Salvador. El caso particular del payaso Chirajito. Apuntes. Revista De Estudios Sobre Patrimonio Cultural, 24(2), 208-221.

Molina-Montecinos, M. (2005). Teatro del oprimido, una herramienta de intervención social (Tesis de pregrado). Universidad Austral de Chile. Facultad de Filosofía y Humanidades. Escuela de Lenguaje y Comunicación. Santiago de Chile, Chile. 
Morgan, D. \& Krueger, R. (1993). When to use focus group and why. En D. Morgan (Ed.) Successful Focus Groups, Advancing the state of the Art. (pp. 3-19). California: Sage Publications.

Murua2.(1990).Ezgaraartistaetaezaktore,saltserobaizik.Argia.Recuperadodehttp:// www.argia.eus/argia-astekaria/1314/xabier-otaegi-eta-jose-ignazio-ansorena.

Nicoll, A. (1977). El mundo de arlequín. Estudio crítico de la Commedia dell' Arte. Barcelona: Barral editores.

Robb, D. (2007). Clowns, Fools and Picaros: Popular Forms in Theatre, Fiction and Film. New York: Rodopi.

Martínez-Rodríguez, J. (2011). Métodos de investigación cualitativa. Silogismo. Revista de Investigación, (8). 1-33.

Rodríguez-Gómez, G., Gil-Flores, J. y García-Jiménez, E. (1996). Metodología de la investigación cualitativa, Málaga: Aljibe.

Ros-Clemente, F.J. (2015a). Cómo reivindicar derechos humanos a través del arte del clown: La función social en el payaso. RES, Revista de educación social, (20). Recuperado de http://www.eduso.net/res/winarcdoc.php?id=530.

Ros-Clemente, F.J. (2015b). La formación de profesionales del clown socioeducativo: una propuesta futura. En A. Fernández-García y Á. De JuanasOliva (Coords.) Pedagogía social, universidad y sociedad (65-71). Madrid: Universidad Nacional de Educación a Distancia (UNED).

Ros-Clemente, F.J., y Úcar, X. (2013). Aportes para la definición, caracterización y expansión de un "clown socioeducativo". En S. Torío-López, O. GarcíaPérez, J.V. Peña-Calvo, C.M. Fernández-García (Coords.) La crisis social y el estado del bienestar: las respuestas de la Pedagogía Social (669-677). España: Servicio de Publicaciones de la Universidad de Oviedo.

Ruch, W., Platt, T. \& Hofmann, J. (2014). The character strengths of class clowns. Frontiers in Psychology, 5, 1-12. Doi: 10.3389/fpsyg.2014.01075.

Sáinz-Moreno, J. (1990). Cuaderno de Circo. Madrid: Gráficas Caro.

Salcedo-Barzola, P., Castro-Salcedo, J.P. y Rivadeneira-González, C.A. (2018). El arte escénico como medio de comunicación: diseño de talleres de cine dirigido a jóvenes - provincia de Santa Elena. Revista Caribeña de Ciencias Sociales, (abril 2018), 1-22. Recuperado de https://www.eumed.net/rev/caribe/2018/04/talleres-cine-jovenes.html. Shepard, B. (2011). Play, Creativity, and Social Movements: If I Can't Dance, It's Not My Revolution. New York: Routledge.

SocialClown. (2014). Los abuelos también ríen. Revista Aarti, (34), 6-7. Recuperado de https://issuu.com/aartiibiza/docs/revista_aarti_1405_vf. Stake, R.E. (1998). Investigación con estudio de casos. Madrid: Morata. Starobinski, J. (2004). Portrait de l'artiste en saltimbanque. París: Gallimard. 
Tójar-Hurtado, J.C. (2001). Planificar la investigación educativa: Una propuesta integrada. Buenos aires: Fundec.

Unanue-Irureta, M. (2012). Muxu eta irrien filosofia. Berria. Recuperado de https://www.berria.eus/paperekoa/1658/046/001/2012-11-25/muxu_eta_ irrien_filosofia.htm.

Vázquez-Recio, R. y Angulo-Rasco, F. (2003). Introducción a los estudios de casos. Los primeros contactos con la investigación etnográfica. Málaga: Aljibe.

Ventosa, V.J. (2008). Perfiles y modelos de Animación y tiempo libre. Madrid: Editorial CCS.

Cómo citar:

Gana-Dañobeitia, A., Huegun-Burgos, A y Rekalde-Rodríguez, I. (2019). Los payasos como herramienta socioeducativa para la intervención comunitaria. Prospectiva. Revista de Trabajo Social e intervención social, (27), 159-186. Doi: $10.25100 /$ prts.v0i27.6733. 